\title{
Cloning and heterologous expression of P450Um-1, a novel bacterial P450 gene, for hydroxylation of immunosuppressive agent AS1387392
}

\author{
Motoi Ueno $^{1}$, Satoshi Sasamura ${ }^{1}$, Midori Yamashita ${ }^{1}$, Nobuharu Shigematsu ${ }^{2}$, Michizane Hashimoto ${ }^{3}$, \\ Motohiro Hino ${ }^{4}$ and Akihiko Fujie ${ }^{5}$
}

Biotransformation technology involving enzymatic modification of original substrates by organisms such as microbes is a valuable tool in improving pharmacokinetics or physicochemical properties of the base compounds. The fungal metabolite AS1387392 is a histone deacetylase inhibitor with potential as a therapeutic immunosuppressant. However, its paucity of functional groups, essential to synthesizing derivatives, is a drawback. Amycolatopsis azurea JCM-3275 catalyzed hydroxylation of AS1387392 to AS1429716, which may facilitate the synthesis of more derivatives by the additional hydroxyl moiety present in AS1429716. This reaction was inhibited by cytochrome P450 inhibitor metyrapone, indicating that cytochrome P450 may be responsible for the transformation. Degenerate PCR primers were subsequently constructed and used to clone genes encoding cytochrome P450 from the genomic DNA of $A$. azurea JCM-3275. We cloned an entire novel P450 gene (1209 bp) and named it P450Um-1. Its deduced amino acid sequence was homologous with that of the CYP105 subfamily. Further cloning of the upstream region, which may contain the native promoter site, was followed by insertion of the open reading frame with the upstream area into Streptomycetes high copy vector plJ702, giving the expression plasmid pNUm-1. P450Um-1 was specifically expressed in Streptomyces lividans TK24, and this recombinant strain converted AS1387392 to AS1429716 without any redox partners. These results show that P450Um-1, a novel bacterial P450, catalyzed hydroxylation of AS1387392 to AS1429716. This resultant recombinant strain is expected to be an efficient biocatalyst with application to more suitable redox systems than those tested here.

The Journal of Antibiotics (2010) 63, 649-656; doi:10.1038/ja.2010.109; published online 6 October 2010

Keywords: Amycolatopsis; AS1429716; CYP105; pIJ702; Streptomyces

\section{INTRODUCTION}

Immunosuppressive agents such as FK506 or cyclosporine have affected therapeutic strategies against acute allograft rejection in organ transplantation procedures. ${ }^{1,2}$ However, side effects such as nephrotoxicity ${ }^{3,4}$ and neurotoxicity ${ }^{5}$ limit their clinical use. To overcome these limitations, a new immunosuppressant based on a novel mechanism must be identified.

Mori et al. ${ }^{6-8}$ reported on the fungal metabolite FR235222 (Figure 1a), an immunosuppressant that works by inhibiting histone deacetylase. The same strain of fungus that produced metabolite FR235222 was also found to produce demethyl-FR235222, known as AS1387392 (Figure 1b). ${ }^{9}$ AS1387392 had an immunosuppressive effect similar to that of FR235222 while maintaining a pharmacokinetic profile approximately two times better than that of FR235222. These findings suggested AS1387392 as a novel immunosuppressive drug candidate for treating acute allograft rejection in organ transplant patients. $^{9}$ However, its paucity of functional groups, essential to synthesizing more derivatives, is a drawback.

Biotransformation is an enzymatic transformation catalyzed by certain organisms, and is often useful in overcoming problems involving biological or physicochemical properties of test compounds. ${ }^{10,11}$ In our search for microorganisms that might catalyze addition of functional groups to AS1387392, we found that Amycolatopsis azurea JCM-3275 hydroxylated the aromatic ring of this compound to form AS1429716 (Figures 1c and 2). ${ }^{12}$ AS1429716 potently inhibited histone deacetylase activity with an $\mathrm{IC}_{50}$ value of $22 \mathrm{nM}$, demonstrating this compound to be as potent as AS1387392 $\left(\mathrm{IC}_{50}=22 \mathrm{~nm}\right)^{9,12}$

Given that several biochemical analyses indicated that cytochrome P450 was responsible for this hydroxylation, we attempted to clone a gene encoding bacterial cytochrome P450 that would catalyze the hydroxylation of AS1387392 to AS1429716. Furthermore,

\footnotetext{
${ }^{1}$ Pharmacology Research Laboratories, Ibaraki, Japan; ${ }^{2}$ Exploratory Research Division, Astellas Research Technologies, Ibaraki, Japan; ${ }^{3}$ Fermentation Research Division, Astellas Research Technologies, Ibaraki, Japan; ${ }^{4}$ Fermentation and Biotechnology Laboratories, Astellas Pharma, Aichi, Japan and ${ }^{5}$ Research Planning \& Administration, Astellas Pharma, Ibaraki, Japan

Correspondence: M Ueno, Pharmacology Research Laboratories, Astellas Pharma, 21 Miyukigaoka, Tsukuba, Ibaraki 305-8585, Japan.

E-mail: motoi.ueno@jp.astellas.com
}

Received 12 February 2010; revised 11 May 2010; accepted 9 September 2010; published online 6 October 2010 


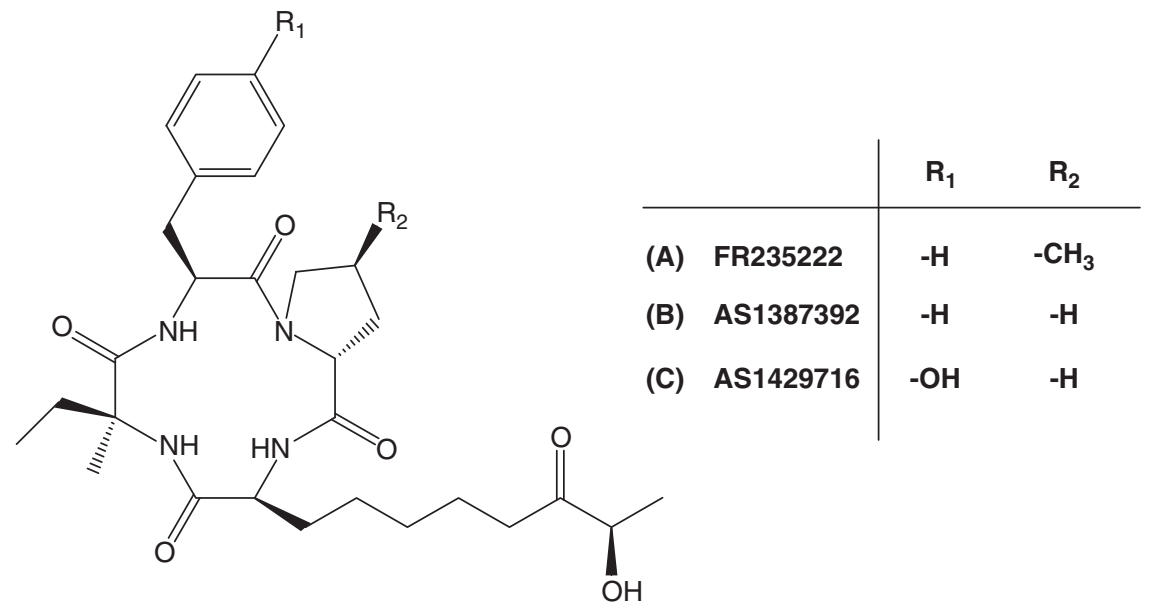

Figure 1 Structure of FR235222 (A), AS1387392 (B) and AS1429716 (C).

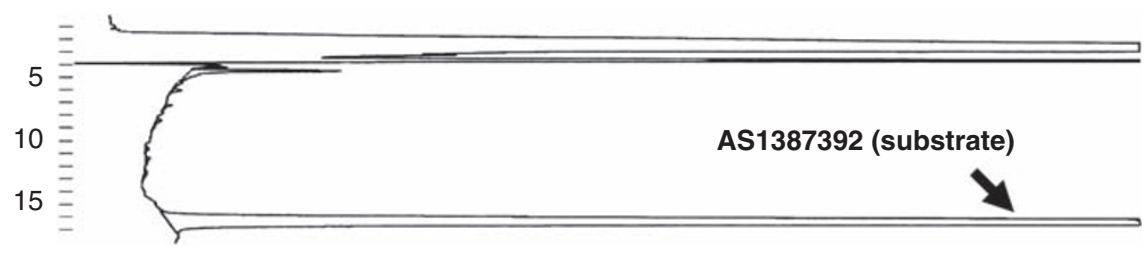

Biotransformation by A. azurea JCM-3275

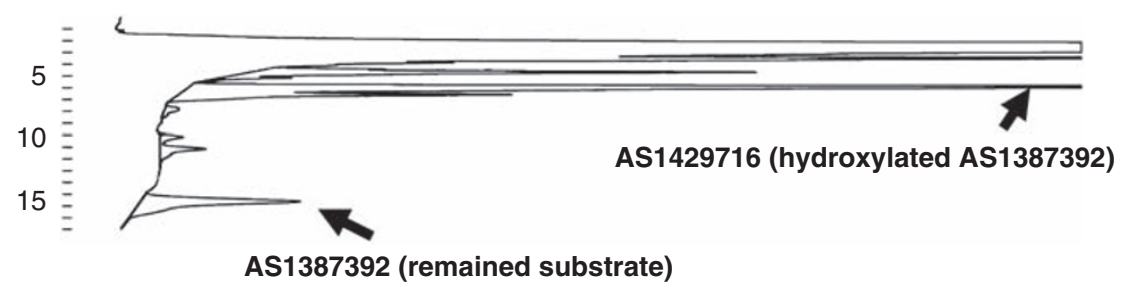

Figure 2 Hydroxylation of AS1387392 to AS1429716 catalyzed by Amycolatopsis azurea JCM-3275. The hydroxylation activity catalyzed by A. azurea JCM3275 was assessed by HPLC. Depicted are the substrate AS1387392 before being mixed with $A$. azurea JCM-3275 (upper chromatogram) and the biotransformed reaction mixture catalyzed by $A$. azurea JCM-3275 (lower chromatogram). Reaction details and HPLC conditions are described in the Materials and methods.

to confirm its function, we attempted to produce its expression in a Streptomycetes host.

\section{MATERIALS AND METHODS \\ Microorganisms}

A. azurea JCM-3275 specimens were purchased from Japan Collection of Microorganisms (Saitama, Japan). Streptomyces lividans TK24 specimens were gifted from the John Innes Centre (Norwich, UK).

\section{Whole-cell biotransformation (hydroxylation) assay (AS1387392 to AS1429716)}

A. azurea JCM- 3275 was inoculated into sterilized seed culture medium ( $\mathrm{pH}$ $7.0,100 \mathrm{ml}$ ) containing glucose $0.5 \%$, sucrose $0.5 \%$, oatmeal (Snow Brand Milk Products, Tokyo, Japan) $0.5 \%$, yeast extract (Becton Dickinson, Franklin Lakes, NJ, USA) $0.2 \%$, peptone (Kyokuto, Tokyo, Japan) $0.5 \%$, peanut powder (Masuda, Chiba, Japan) $0.5 \%$, humic acid (IIC, Chiba, Japan) $0.01 \%$, Tween- $800.1 \%$ and $\mathrm{CaCO}_{3} 0.2 \%$ in $225 \mathrm{ml}$ Erlenmeyer flasks. The inoculated flask was shaken on a rotary shaker $\left(220\right.$ r.p.m., $5.1 \mathrm{~cm}$ throw) at $30^{\circ} \mathrm{C}$ for 3 days. The seed culture (2\%) was inoculated into sterilized expression culture medium (pH 6.5, $100 \mathrm{ml}$ ) containing glycerol 6.0\%, soybean flour (Matsumoto,
Osaka, Japan) $1.0 \%$ and corn steep liquor (Nihon Shokuhin Kako, Tokyo, Japan) $3.0 \%$ in $225 \mathrm{ml}$ Erlenmeyer flasks. Expression culture was conducted at $30^{\circ} \mathrm{C}$ for 3 days on a rotary shaker ( 220 r.p.m., $5.1 \mathrm{~cm}$ throw).

The biotransformation reaction was initiated by addition of AS1387392 (final concentration, $500 \mu \mathrm{g} \mathrm{m}^{-1}$ ) to the broth. After a further $12 \mathrm{~h}$ of incubation, an equal amount of acetone was added to the mixture to stop reaction progression. The broth was centrifuged to obtain the supernatant $(5000 \times \mathrm{g}$, $10 \mathrm{~min}$ ), which was extracted using an equal volume of ethyl acetate. The resultant extract was evaporated and dissolved with methanol.

To monitor the hydroxylated compound AS1429716, LC/MS analysis was performed on the extracted sample under the following conditions: the system was an Agilent HP1100 series LC/MSD (Hewlett-Packard, Palo Alto, CA, USA), the column was an Xterra MS C18 $(2.1 \times 20 \mathrm{~mm}, 2.5 \mu \mathrm{m})$, the mobile phase was $30 \%$ acetonitrile, the flow rate was $0.2 \mathrm{ml} \mathrm{min}^{-1}$, the temperature was $50^{\circ} \mathrm{C}$, the fragmentor voltage was $160 \mathrm{~V}$ and the analytical mode was selected-ion monitoring mode that allowed for specific, sensitive and quantitative detection of AS1429716-related compounds. Each retention time of AS1387392 and AS1429716 was 5.3 and $1.9 \mathrm{~min}$, respectively. Conditions for HPLC analysis were as follows: the system was a D-7000 HPLC system (Hitachi, Tokyo, Japan), the column was a YMC ODS-AM AM-303 $(250 \times 4.6 \mathrm{~mm}, 5 \mu \mathrm{m})$, the mobile 
phase was $40 \%$ acetonitrile, the flow rate was $1.0 \mathrm{ml} \mathrm{min}^{-1}$, the temperature was $50{ }^{\circ} \mathrm{C}$ and the absorbance was $210 \mathrm{~nm}$. Each retention time of AS1387392 and AS1429716 was 16.1 and $5.9 \mathrm{~min}$, respectively.

\section{Biotransformation assay under cell-free conditions with A. azurea} JCM-3275 lysate

The mycelium of A. azurea JCM-3275 was resuspended in $50 \mathrm{~mm}$ sodium phosphate buffer ( $\mathrm{pH} 7.4$ ) containing $1 \mathrm{~mm}$ dithiothreitol, $20 \%$ glycerol, $0.7 \mu \mathrm{g} \mathrm{ml}^{-1}$ pepstatin, $0.5 \mu \mathrm{g} \mathrm{ml}^{-1}$ leupeptin and $0.2 \mathrm{~mm}$ phenylmethylsulfonyl fluoride. The suspension was sonicated on ice with an ultrasonic processor at $60 \%$ of the maximum amplitude for $30 \mathrm{~s}$, and then centrifuged at $100000 \times \mathrm{g}$ for $60 \mathrm{~min}$ to remove cell debris and membranes. The obtained supernatant was the lysate of A. azurea JCM-3275.

To start the biotransformation reaction, the lysate was supplemented with $1 \mathrm{~mm} \mathrm{NADH}, 0.8 \mathrm{mg} \mathrm{ml}^{-1}$ ferredoxin (from spinach; Sigma-Aldrich, St Louis, MI, USA), 0.2 units $\mathrm{ml}^{-1}$ ferredoxin-NADP ${ }^{+}$reductase (from spinach; SigmaAldrich) and the substrate AS1387392 $\left(500 \mu \mathrm{g} \mathrm{ml}^{-1}\right)$. Detection and quantification of the converted product AS1429716 was carried out by LC/MS as described above. To evaluate its inhibitory effect, P450 inhibitor metyrapone (Sigma-Aldrich) was added to the reaction system at various concentrations.

\section{Cloning of P450 gene fragment from A. azurea JCM-3275}

To clone the $\mathrm{P} 450$ gene fragment (approximately $600 \mathrm{bp}$ ), degenerate primers were constructed (forward, 5'-TTCGCSCTSCCSGTSCC-3'; reverse, 5'-GC TGBCCSAGGCACTGGT-3'). PCR was performed using Amplitaq Gold (Applied Biosystems, Tokyo, Japan) on a Perkin-Elmer DNA thermal cycler (Yokohama, Japan). Time and temperature conditions were as follows: activation of polymerase at $95^{\circ} \mathrm{C}$ for $10 \mathrm{~min}, 40$ cycles of denaturation at $94{ }^{\circ} \mathrm{C}$ for $30 \mathrm{~s}$, annealing and extension at $60^{\circ} \mathrm{C}$ for $30 \mathrm{~s}$ and extension at $60^{\circ} \mathrm{C}$ for $10 \mathrm{~min}$.

The amplified DNA fragment was ligated to a pGEM T-easy cloning vector (Promega, Madison, WI, USA) according to the manufacturer's protocol and subsequently introduced into Escherichia coli JM109 (Takara Bio, Shiga, Japan). A plasmid was prepared from an ampicillin-resistant clone using a QIAprep Spin Miniprep Kit (Qiagen, Hilden, Germany), and nucleotide sequencing was subsequently conducted using an ABI PRISM 3100 Genetic Analyzer (Applied Biosystems), which included conducting sequence analyses with newly constructed primers toward adjacent un-sequenced regions in already-sequenced regions

\section{Construction of genomic cosmid library of A. azurea JCM-3275} Genomic DNA of $A$. azurea JCM-3275, prepared using a QIAGEN Genomictip System (Qiagen), was partially digested with an appropriate concentration of Sau3AI to produce DNA fragments approximately $40 \mathrm{~kb}$ in length (size verified by agarose gel $(0.4 \%)$ electrophoresis). The obtained DNA fragments were ligated to a SuperCos cosmid vector (Stratagene, La Jolla, CA, USA) and packed into a $\lambda$ phage using Gigapack III XL packaging extract (Stratagene) by the recommended protocol. XL-1 Blue MRF' (Stratagene) was transformed with the recombinant phage, being resistant against $100 \mu \mathrm{g} \mathrm{ml}^{-1}$ of ampicillin. The cosmid was prepared from the resultant recombinant clone, through the same procedure as that used for plasmid preparation.

\section{PCR screening to identify clones with an entire P450 gene, and sequence analysis of the positive cosmid}

To screen clones possessing an entire P450 gene, authentic primers (forward, 5'-TTCGCGCTGCCGGTGCC-3'; reverse, 5'-GCTGGCCGAGGCACTGGT-3') were constructed. PCR was performed with these primers and the obtained cosmids under the same conditions as described above. The cosmid from which the DNA fragment (expected size $\sim 600 \mathrm{bp}$ ) was amplified was designated the positive cosmid. To clarify the whole $\mathrm{P} 450$ open reading frame, sequence analysis of the positive cosmid was conducted using the primer walking method. The region upstream of the open reading frame was also sequenced (approximately $850 \mathrm{bp}$ ). Amino acid comparison was carried out between the translated sequence and already-known proteins (CYP105C1 and CYP105D1) registered in the Cytochrome P450 Homepage database (http://drnelson.uthsc. edu/CytochromeP450.html) using either the BLAST algorithm or Genetyx software (Osaka, Japan).
Heterologous expression of the P450Um-1 gene in S. lividans TK24 The P450Um-1 gene and the upstream region, possibly including the native promoter site, were amplified together using PCR with appropriate primers and the positive cosmid. To clone the targeted site, PCR primers with an additional restriction site (a KpnI site at the $5^{\prime}$-end and a SacI site at the $3^{\prime}$-end) were constructed. PCR was then performed using Pfu polymerase (Stratagene) at $96^{\circ} \mathrm{C}$ for $1 \mathrm{~min}, 66.6^{\circ} \mathrm{C}$ for $2 \mathrm{~min}$ and $74^{\circ} \mathrm{C}$ for $5 \mathrm{~min}$ for amplification $(30$ cycles), and $74^{\circ} \mathrm{C}$ for $7 \mathrm{~min}$ for extension ( 1 cycle). The amplified PCR products were sequenced to confirm no errors in the PCR had occurred, and were then digested with $\mathrm{KpnI} / \mathrm{SacI}$ and subsequently ligated into a previously KpnI/SacI-digested pIJ702, a high-copy-number cloning vector for use with Stretptomyces spp. ${ }^{13}$ The resultant expression plasmid pNUm-1 was introduced into S. lividans TK24 by the standard procedure. ${ }^{14}$ Transformants containing the targeted fragment were selected from thiostrepton-resistant colonies.

The mycelium of $S$. lividans TK24 harboring pNUm-1 was inoculated into sterilized seed culture medium $(100 \mathrm{ml})$ containing glucose $0.5 \%$, sucrose $0.5 \%$, oatmeal (Snow Brand Milk Products) 0.5\%, yeast extract (Becton Dickinson) $0.2 \%$, peptone (Kyokuto) 0.5\%, peanut powder (Masuda) $0.5 \%$, humic acid (IIC) $0.01 \%$, Tween- $800.1 \%, \mathrm{CaCO}_{3} 0.2 \%$ and thiostrepton $5 \mu \mathrm{g} \mathrm{m}^{-1}(\mathrm{pH}$ adjusted to 7.0 ) in $225 \mathrm{ml}$ Erlenmeyer flasks. The inoculated flasks were shaken on a rotary shaker $\left(240\right.$ r.p.m.) at $30^{\circ} \mathrm{C}$ for 3 days, and then inoculated $(2 \%)$ into sterilized expression culture medium yeast extract-malt extract $(100 \mathrm{ml})$ containing glucose $1.0 \%$, yeast extract (Becton Dickinson) $0.3 \%$, malt extract (Becton Dickinson) $0.3 \%$, peptone (Kyokuto) $0.5 \%$, sucrose $34 \%$ and $\mathrm{MgCl}_{2} 6 \mathrm{H}_{2} \mathrm{O} \quad 0.05 \%$ in $225 \mathrm{ml}$ Erlenmeyer flasks. The flasks were incubated on a rotary shaker ( 240 r.p.m.) at $30^{\circ} \mathrm{C}$ for 3 days. After cultivation, the mycelium was subjected to either SDS-polyacrylamide gel electrophoresis analysis or a biotransformation assay.

\section{SDS-polyacrylamide gel electrophoresis analysis and MASCOT search}

Samples for SDS-polyacrylamide gel electrophoresis analysis were prepared by subjecting mycelium collected from approximately $100 \mu \mathrm{l}$ of broth to sonication and centrifugation $(10000 \times g, 10 \mathrm{~min})$. The supernatant samples, with protein levels adjusted to equal quantities, were applied to the gel. After electrophoresis, the gel was stained with Coomassie Brilliant Blue R-250 (Bio-Rad, Tokyo, Japan).

The gel was cut away and incubated with acetonitrile, after which the solvent was evaporated. After bleaching with $100 \mathrm{mM} \mathrm{NH}_{4} \mathrm{HCO}_{3}$ and acetonitrile, the gel was soaked in $10 \mathrm{~mm}$ dithiothreitol and $100 \mathrm{mM} \mathrm{NH}_{4} \mathrm{HCO}_{3}$ and incubated at $56^{\circ} \mathrm{C}$ for $45 \mathrm{~min}$. Following removal of the supernatant, $55 \mathrm{~mm}$ iodoacetamide in $100 \mathrm{mM} \mathrm{NH}_{4} \mathrm{HCO}_{3}$ was added. The iodoacetamide was then removed, and the gel was washed with $100 \mathrm{~mm} \mathrm{NH}_{4} \mathrm{HCO}_{3}$. The gel was then placed in trypsin buffer containing trypsin $12.5 \mu \mathrm{g} \mu \mathrm{l}^{-1}, \mathrm{CaCl}_{2} 5 \mathrm{~mm}$ and $\mathrm{NH}_{4} \mathrm{HCO}_{3}$ $50 \mathrm{~mm}$, and incubated on ice for $25 \mathrm{~min}$. The trypsin buffer was removed, and incubation buffer $\left(\mathrm{CaCl}_{2}(5 \mathrm{~mm})\right.$ and $\left.\mathrm{NH}_{4} \mathrm{HCO}_{3}(50 \mathrm{~mm})\right)$ was added, in which the gel was incubated at $37^{\circ} \mathrm{C}$ for over $12 \mathrm{~h}$. The trypsin-digested peptide fragment solution was used in matrix-assisted laser desorption/ionization-time-of-flight MS analysis with a Micromass TofSpec 2E (Micromass, Manchester, UK) to identify peptides using Mascot database search algorithms. Dihydroxy benzene was used as the matrix.

The cover ratio was defined as the proportion of peptide fragments corresponding to $P 450 U m-1$ to the total number of detected fragments. The deduced amino acid sequence of P450Um-1 was registered in advance with the concerned database.

\section{Whole-cell biotransformation using S. lividans TK24 expressing P450Um-1}

S. lividans TK24-expressing pNUm-1 was grown as described above. The substrate, AS1387392 (final concentration, $500 \mu \mathrm{g} \mathrm{m}^{-1}$ ), was added to the broth culture to initiate the biotransformation reaction. The reaction mixture was incubated at $30^{\circ} \mathrm{C}$ on a rotary shaker $(240$ r.p.m.) for $12 \mathrm{~h}$, and a recombinant strain containing pIJ702 alone was employed as a negative control. To stop reaction progression at the end of incubation, acetone was added to the reaction mixture, followed by centrifugation to acquire the supernatant for monitoring the converted compound AS1429716 by the 
LC/MS method described above. Mass spectrum analyses with the peaks specifically detected in the expression of P450Um-1 were performed with Agilent HP1100 series LC/MSD (Hewlett-Packard),

\section{RESULTS}

\section{Hydroxylation of AS1429716 by A. azurea JCM-3275}

Cytochrome P450, a heme-containing enzyme present in various organisms, ${ }^{15}$ is known to cause hydroxylation in several kinds of substrates. Given this property, we suspected that cytochrome P450 may be responsible for the hydroxylation catalyzed by A. azurea JCM3275. To test this hypothesis and assess whether the P450 inhibitor, metyrapone, ${ }^{16}$ inhibited hydroxylation activity, we constructed a cell-free reaction system. This system contained cell lysate supplemented with NADPH, ferredoxin, ferredoxin reductase and substrate AS1387392. The converted product AS1429716 (molecular weight $=558 \mathrm{~g} \mathrm{~mol}^{-1}$ ) was detected by LC/MS, which showed that the protonic peak $\left(\mathrm{M}+\mathrm{H}^{+} ; 559\right)$ was dominant. Given these findings, we set the selected ion monitoring mode to detect AS1429716 specifically, sensitively and quantitatively; amount of AS1429716 was calculated in this mode. Metyrapone was found to dose-dependently inhibit the reaction (Figure 3), suggesting that the hydroxylation activity exhibited by A. azurea JCM-3275 is indeed attributed to cytochrome P450.

\section{Cloning the P450 gene from A. azurea JCM-3275}

We suspected that the oxidative reaction in which AS1387392 is converted to AS1429716 was catalyzed by cytochrome P450. One method of confirming whether this enzyme actually participates in this oxidative reaction is to clone candidate $\mathrm{P} 450$ genes from $A$. azurea JCM-3275, which are homologous to the bacterial P450 gene, and verify the function of the responsible gene.

Whole-cell biotransformation utilizing the recombinant bacterial strains in which the gene is heterologously expressed may be economical in functional analyses of the P450 gene, as the resultant strains may also be useful as biocatalysts. Therefore, we attempted to clone and heterologously express the gene involved in AS1387392 hydroxylation.

Given that many P450 enzymes belonging to the CYP105 subfamily are derived from Actinomycetes and oxidize a wide array of substrates, ${ }^{17,18}$ we constructed degenerate PCR primers founded on the conserved region within the CYP105 subfamily. PCR was performed

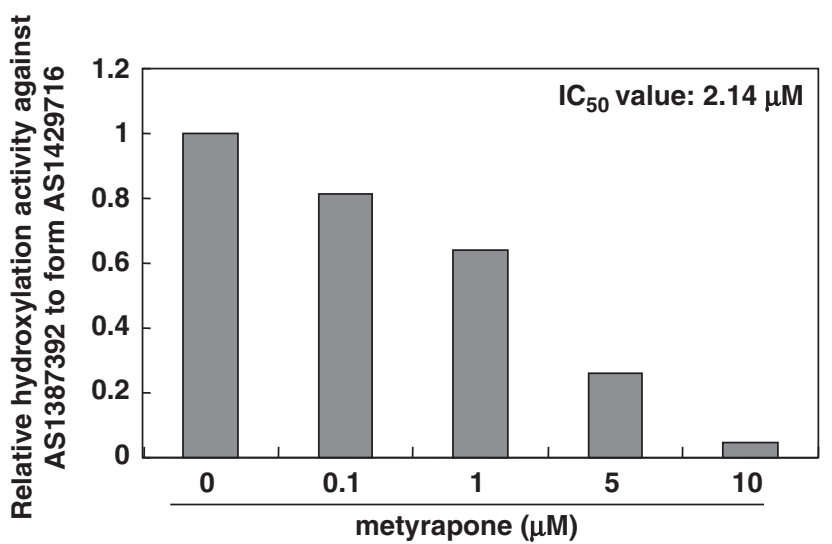

Figure 3 Inhibitory effect of cytochrome P450 inhibitor metyrapone on the hydroxylation of AS1387392 to AS1429716 in the cell-free system with Amycolatopsis azurea JCM-3275 lysate. Hydroxylation activity was analyzed by LC/MS, in which the hydroxylated compound AS1429716 could be quantitatively detected. using genomic DNA from A. azurea JCM-3275, and an amplified fragment (approximately $600 \mathrm{bp}$ ) was obtained. Subsequent sequence analysis demonstrated that the amplified DNA was a part of cytochrome P450.

The A. azurea JCM-3275 genome DNA fragments that had been partially digested with Sau3AI to roughly $40 \mathrm{kbp}$ were inserted into the cosmid vector SuperCos, and then packaged into E. coli XL-1 Blue MRF' to provide a genomic DNA library of A. azurea JCM-3275. To obtain positive clones with the entire cytochrome $\mathrm{P} 450$ from which the partial fragment was cloned, PCR screening using authentic primers reconstructed from the confirmed sequence of the amplified DNA fragments was conducted. Screening provided two positive clones, from which we were able to clone the novel entire cytochrome P450 gene (1209 bp) through primer walking analysis.

This P450 gene was named the P450Um-1 gene and encoded an oxygen binding and a heme-iron binding region, both of which were typical motifs of cytochrome P450 (Figure 4). These sequence data have been submitted to the DDBJ (DNA Data Bank of Japan) databases under accession no. AB208694. Comparison of the deduced amino acid sequence of $P 450 U m-1$ with that of $\mathrm{CYP} 105 \mathrm{Cl}^{19}$ and CYP $105 D 1^{17}$ revealed 48 and $42 \%$ similarity, respectively (Figure 5). Thus, according to the $40 \%$ rule, which states that two different P450 enzymes belong to the same subfamily if their amino acid sequences are $\geqslant 40 \%$ similar, P450Um- 1 was designated as a new member of the CYP105 subfamily. ${ }^{20}$

\section{Heterologous expression of P450Um-1 gene in S. lividans TK24}

To further examine its function, we attempted to express the P450Um-1 gene. Given the high GC content of the P450Um-1 gene (67.8\%), the genus Streptomycetes appeared a suitable host to functionally express the gene. We also cloned the upstream region (718bp), which likely included the native promoter site, often located several hundred bp ahead of the open reading frame in Actinomycetes. ${ }^{21}$

The obtained fragment (P450Um-1 gene and the upstream region) was inserted into Streptomycetes cloning vector pIJ702, in which no inducible promoter was present. The resultant expression plasmid, pNUm-1, was introduced into S. lividans TK24 to induce expression of the P450Um-1 gene. The transformants, resistant toward thiostrepton, were grown in yeast extract-malt extract liquid medium at $30{ }^{\circ} \mathrm{C}, 240$ r.p.m., for 3 days under the assumption that the native promoter should spontaneously trigger expression of $P 450 \mathrm{Um}-1$ without any inducers.

After SDS-polyacrylamide gel electrophoresis analysis and staining the lysates prepared from the transformants with Coomassie Brilliant Blue, we extracted proteins with molecular weights near $45 \mathrm{kDa}$ (expected size of $P 450 U m-1=44.8 \mathrm{kDa}$ ) and digested them with trypsin. After registering amino acid sequence information of $P 450 U m-1$ on the concerned database in advance, the obtained peptide fragments were subjected to matrix-assisted laser desorption/ionization-time-of-flight MS analysis, leading to the observation that a number of peptide fragments from the recombinant strain harboring $\mathrm{pNUm}-1$ were attributed to P450Um-1 (Figure 6). The obtained data were analyzed by a Mascot database search algorithm. Given that the cover ratio of P450Um-1 was $61 \%$, whereas that in the negative control strain harboring pIJ702 was $0 \%$, we concluded that $P 450 U m$ - 1 was specifically expressed in S. lividans TK24 harboring pNUm-1.

\section{Hydroxylation of AS1387392 to AS1429716 by S. lividans TK24 harboring pNUm-1}

Usually, an appropriate electron transfer partner must be coexpressed with the P450 gene to oxidize a foreign substrate. ${ }^{22}$ However, we 


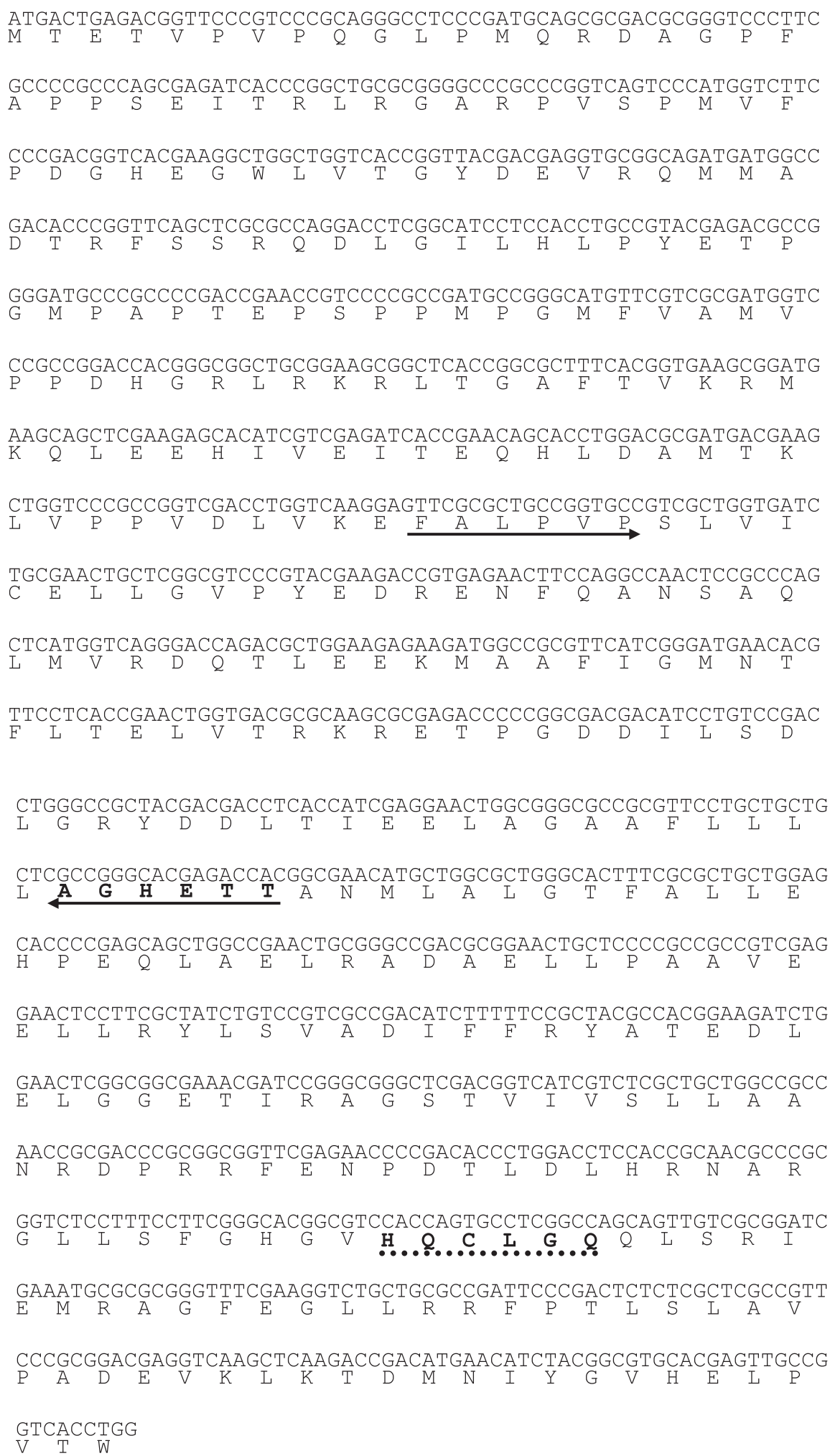

Figure 4 Nucleotide sequence (1209 bp) and deduced amino acid sequence of P450Um-1, a novel bacterial P450 from Amycolatopsis azurea JCM-3275. Degenerate primers were constructed based on the region under which the arrows are drawn in the figure. The area indicated by the arrow facing left is the oxygen-binding motif, and the motif on the dotted line is the heme-iron-binding motif. Both motifs are typical scaffolds of cytochrome P450. These sequence data have been submitted to the DNA Data Bank of Japan (DDBJ) databases under accession no. AB208694. 


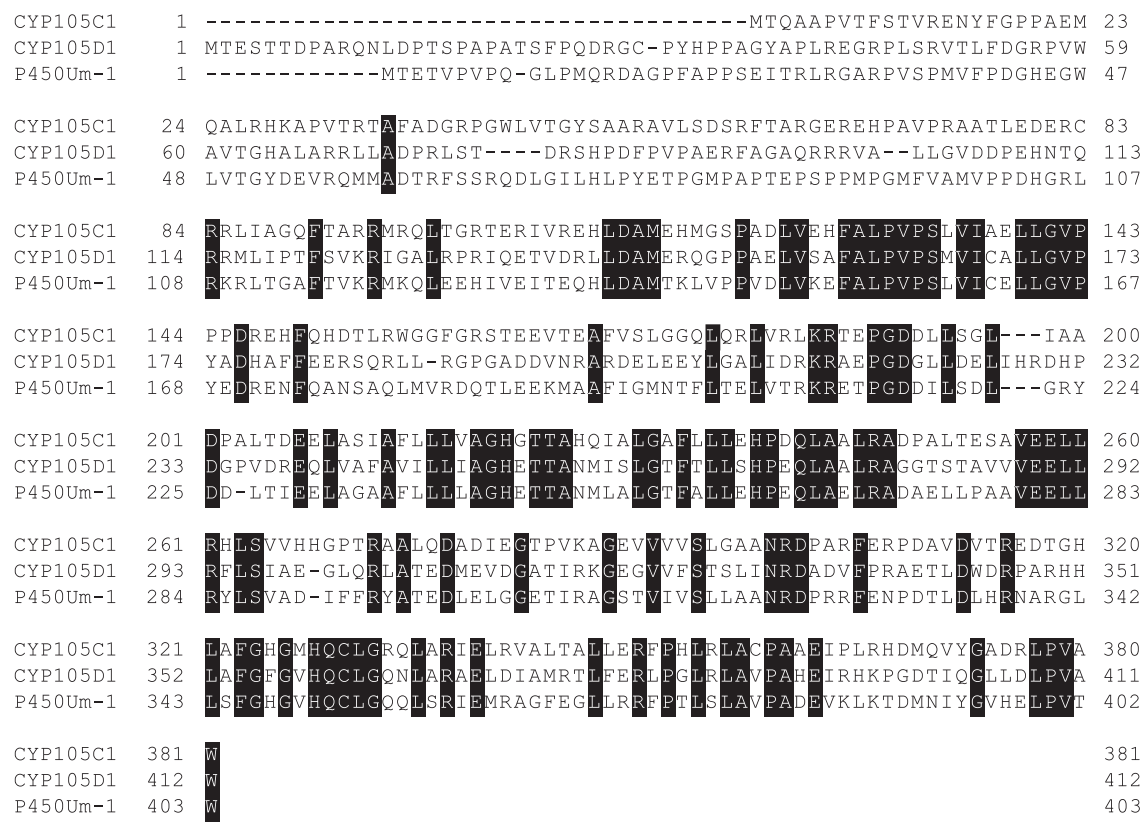

Figure 5 A comparison of deduced amino acid sequence of P450Um-1 with that of CYP105C1 and CYP105D1. Alignment was carried out by Genetyx software. Identical regions among the three P450 proteins are denoted by the enclosed box. The amino acid sequences of CYP105C1 and CYP105D1 are quoted from the Cytochrome P450 homepage (http://drnelson.uthsc.edu/CytochromeP450.html).

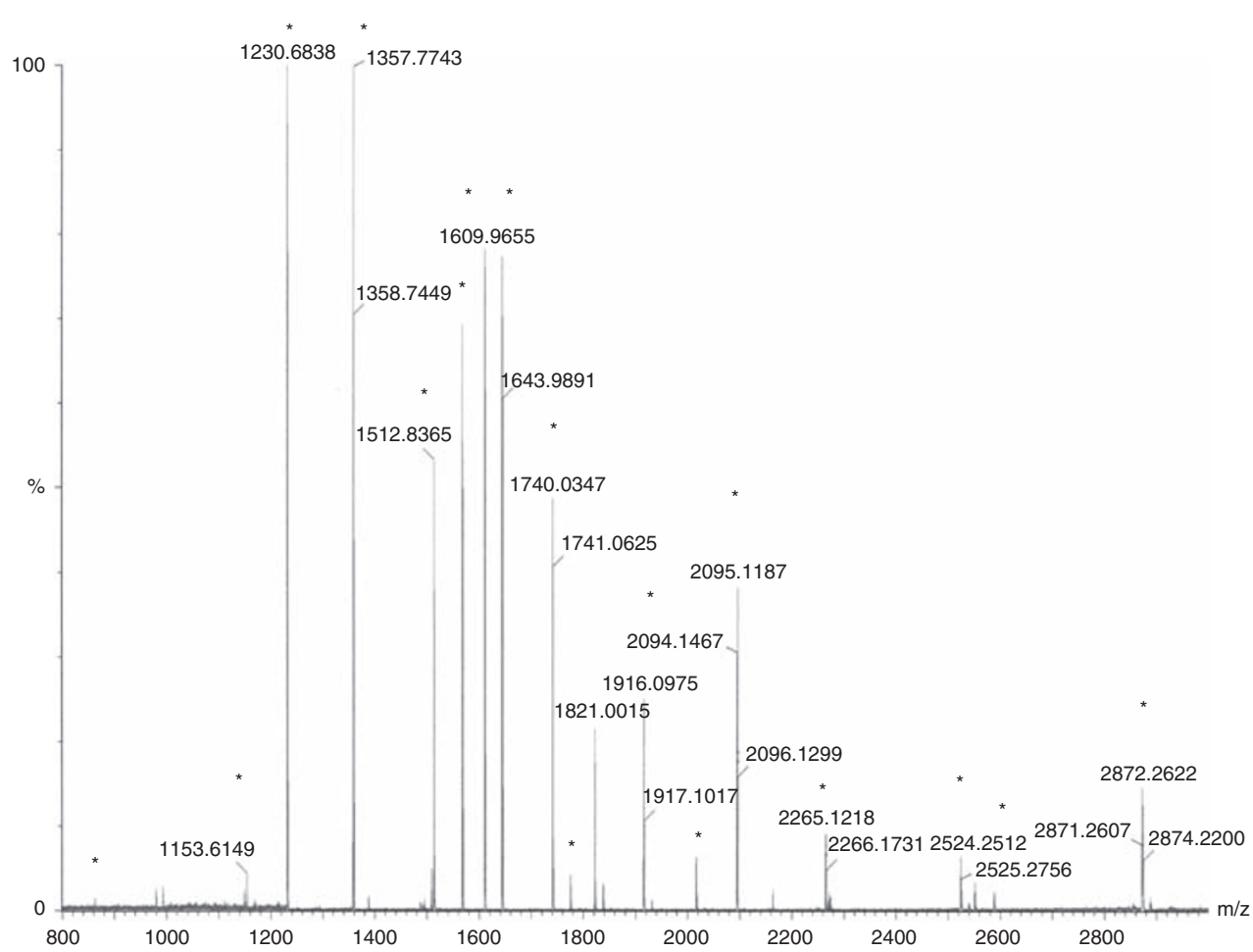

Figure 6 Matrix-assisted laser desorption/ionization-time-of-flight MS (MALDI/TOF-MS) analysis of peptide fragments prepared from Streptomyces lividans TK24 harboring pNUm-1. Peaks with asterisks over them are attributed to P450Um-1. Dihydroxy benzene (DHB) was used as the matrix.

attempted to induce expression of the P450Um-1 gene alone, on the assumption that an endogenous redox partner in the host may improve oxidative activity. Whole-cell biotransformation of AS1387392 to AS1429716 was performed using the recombinant strain expressing P450Um-1. AS1387392 was added to the broth after $72 \mathrm{~h}$ of expression culture, and the reaction was conducted for a further $12 \mathrm{~h}$. The reaction was stopped by adding acetone to the biotransformation mixture, and the converted product AS1429716 was detected by LC/MS as described above. When P450Um-1 was expressed, we were able to specifically detect three peaks, which we named 'peak $X$ ', 


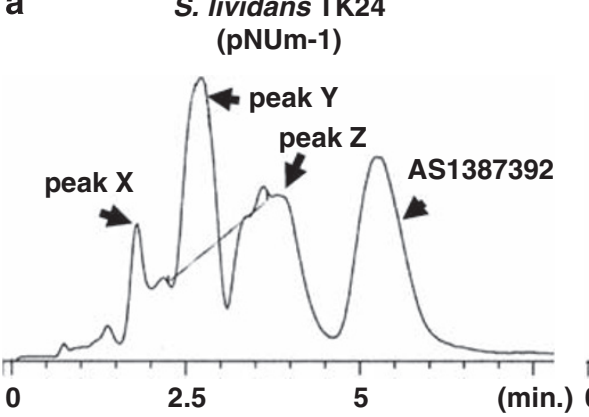

S. lividans TK24

(plJ702)

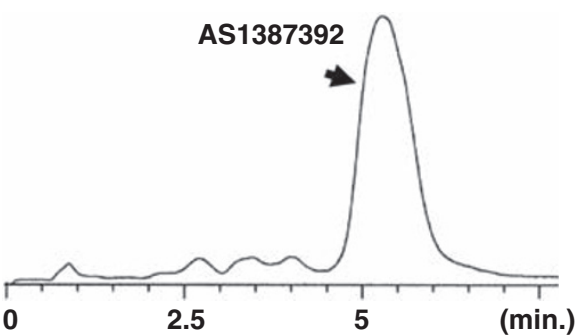

b

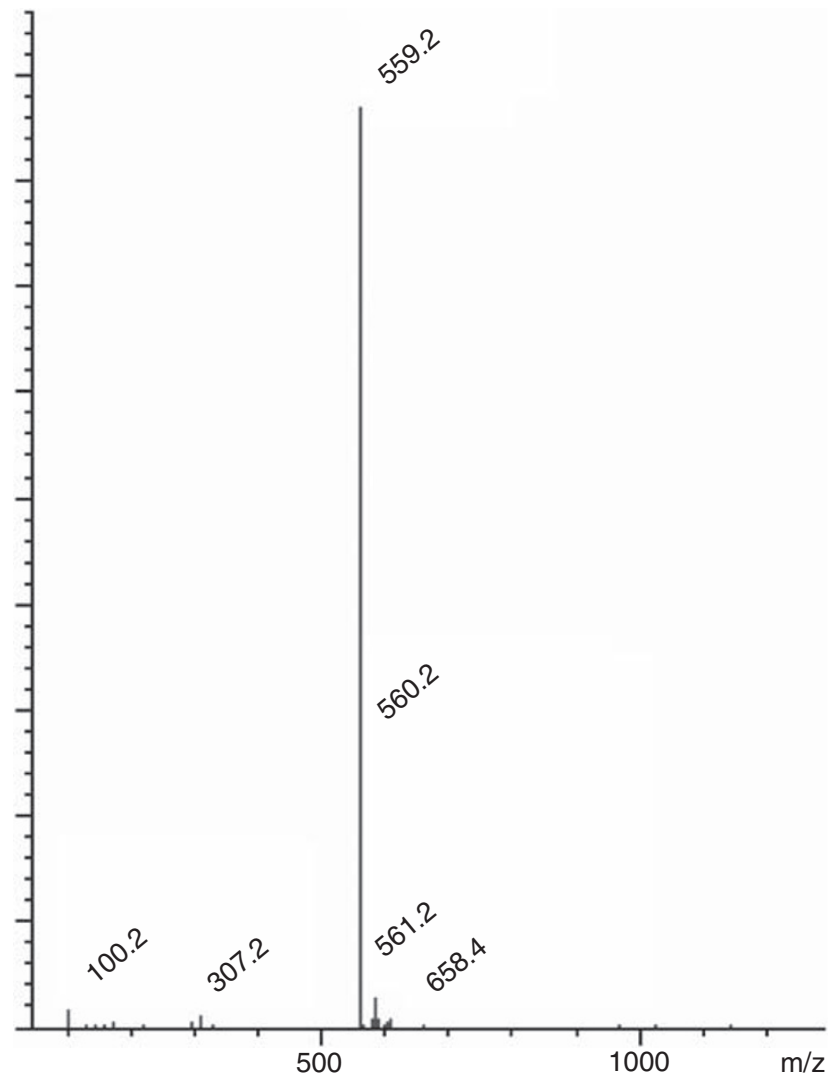

Figure 7 (a) Bioconversion reactions of AS1387392 by Streptomyces lividans TK24 expressing P450Um-1. The converted products were detected by LC/MS. Details regarding conditions and methods are described in the Materials and methods section. The bioconversion of AS1387392 as catalyzed by S. lividans TK24 harboring pNUm-1 is shown in the left panel, whereas no reaction is seen in the right panel, showing pIJ702 alone. Three peaks that were specifically detected in the expression of P450Um-1 were named 'peak X', 'peak $Y$ ' and 'peak Z', respectively. (b) Mass spectrum of the converted product 'peak X' specifically detected in the expression of P450Um-1. This mass spectrum agreed with that of AS1429716 with the dominant protonic peak $\left(\mathrm{M}+\mathrm{H}^{+} ; 559\right)$.

'peak Y' and 'peak Z', respectively (Figure 7a). The mass spectrum of the 'peak X' coincided with that of AS1429716 (molecular weight $\left.=558 \mathrm{~g} \mathrm{~mol}^{-1}\right)$, in which the protonic peak $\left(\mathrm{M}+\mathrm{H}^{+} ; 559\right)$ was

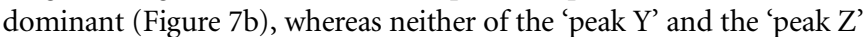
could be identified (data not shown). Given these observations, we concluded that P450Um-1 was closely involved in hydroxylation of AS1387392, and that the electron transfer system from S. lividans TK24 was functioning, at least in part.

\section{DISCUSSION}

Microbial biocatalysts often specifically hydroxylate inactive sites, such as a methylene moiety, ${ }^{23,24}$ which facilitates synthesis of more derivatives from the resultant hydroxyl moiety. With its aromatic ring hydroxylated by $A$. azurea JCM-3275 to add the active moiety, AS1429716 is a novel immunosuppressive drug candidate. Therefore, identification of an enzyme able to catalyze this biotransformation reaction and create an efficient production system would be extremely useful. We hypothesized that this reaction may have been accomplished by the cytochrome P450 enzyme, because administration of the P450 inhibitor metyrapone at $10 \mu \mathrm{M}$ completely inhibited the reaction. The Actinomycetes P450 enzymes identified so far belong to one of several subfamilies, ${ }^{25,26}$ and members of the CYP105 subfamily, originated from Actinomycetes, specifically catalyze oxidative reactions on various substrates, which motivated us to clone CYP105 gene specifically. The 'FALPVP' motif, in which the degenerate PCR primer was founded, is the conserved region of the CYP105 subfamily just 
only. In the present study, P450Um-1 is the only gene that we could clone with the primers employed in our research. We did not try to examine another primer sets, as we gave the functional analysis of P450Um-1 the highest priority.

To investigate whether P450Um-1 was involved in the hydroxylation of AS1387392 to AS1429716, we attempted to induce heterologous expression of P450Um-1. First, we inserted the P450Um-1 gene into the thiostrepton-inducible Streptomyces expression vector pIJ602127 and attempted to induce expression in S. lividans TK24. However, P450Um-1 was not produced under any conditions tested, an observation probably because of the unsuitability of the expression system for P450Um-1 (data not shown).

Cytochrome P450 enzymes are usually coupled with a redox partner to function properly. However, given that bacterial P450 expressed alone in heterologous host has been reported to function with the assistance of endogenous redox partners, ${ }^{28}$ we conducted whole-cell biotransformation using the recombinant strain harboring P450Um-1 that lacked coexpression of any foreign redox partners. The observed hydroxylation may have been facilitated by the endogenous electron transfer system in S. lividans TK24. The present findings demonstrate that P450Um-1, a novel bacterial P450, catalyzed hydroxylation of AS1387392 to AS1429716.

Although biotransformation activity was detected, the conversion ratio was not markedly high. Previous studies achieved effective biotransformation with coexpression of CYP105A1 and CYP105B1 and appropriate redox components in S. lividans $1326 .{ }^{29}$ In addition, the $\mathrm{P}_{\text {nitA }}$-NitR system, a hyperinducible expression scheme, proved useful for expressing various kinds of enzymes. ${ }^{30}$ Based on these previous findings, good electron transfer partners and expression systems for the P450Um-1 gene may need to be identified in order to utilize the gene's intrinsic potential. Moreover, P450Um-1 is a novel member of the CYP105 subfamily, and thus may respond to other substrates in a manner similar to CYP105D $1,{ }^{17}$ which oxidizes a wide array of substrates. Therefore, it is significant to clarify the unidentified peaks detected in the conversion of AS1387392.

Further studies are necessary to produce the desired activities of heterologously expressed cytochrome P450 in a whole-cell transformation assay. Construction of universal expression systems for bacterial cytochrome $\mathrm{P} 450$ as an effective biocatalyst will greatly impact both the industrial and academic spheres.

1 First, M. R. Immunosuppressive agents and their actions. Transplant. Proc. 34, 1369-1371 (2002).

2 Gorantla, V. S. et al. Immunosuppressive agents in transplantation: mechanism of action and current anti-rejection strategies. Microsurgery 20, 420-429 (2000).

3 Su, Q., Weber, L., Hir, M. L., Zenke, G. \& Ryffel, B. Nephrotoxicity of cyclosporin A and FK506: inhibition of calcineurin phosphatase. Ren. Physiol. Biochem. 18, 128-139 (1995).

4 Mcdiarmid, S. V. et al. A comparison of renal function in cyclosporine- and FK506-treated patients after primary orthotopic liver transplantation. Transplantation 56, 847-853 (1993)
5 Bechstein, W. O. Neurotoxicity of calcineurin inhibitors: impact and clinical management. Transpl. Int. 13, 313-326 (2000).

6 Mori, H. et al. FR235222, a fungal metabolite, is a novel immunosuppressant that inhibits mammalian histone deacetylase (HDAC). I. Taxonomy, fermentation, isolation and biological activities. J. Antibiot. 56, 72-79 (2003).

7 Mori, H. et al. FR235222, a fungal metabolite, is a novel immunosuppressant that inhibits mammalian histone deacetylase (HDAC). II. Biological activities in animal models. J. Antibiot. 56, 80-86 (2003).

8 Mori, H. et al. FR235222, a fungal metabolite, is a novel immunosuppressant that inhibits mammalian histone deacetylase (HDAC). III. Structure determination. J. Antibiot. 56, 181-185 (2003).

9 Sasamura, S. et al. AS1387392, a novel immunosuppressive cyclic tetrapeptide compound with inhibitory activity against mammalian histone deacetylase. J. Antibiot. (e-pub ahead of print 30 June 2010; doi:10.1038/ja.2010.51).

10 Dordick, J. S. \& Clark, D. S. Biocatalysis and biotransformation. Curr. Opin. Chem. Biol. 6, 123-124 (2002).

11 Machida, K., Aritoku, Y., Nakashima, T., Arisawa, A. \& Tsuchida, T. Increase in pladienolide D production rate using a Streptomyces strain overexpressing a cytochrome P450 gene. J. Biosci. Bioeng. 105, 649-654 (2008).

12 Sasamura, S. et al. Bioconversion of AS1387392: bioconversion studies involving Amycolatopsis azurea JCM 3275. J. Antibiot. (e-pub ahead of print 6 October 2010; doi:10.1038/ja.2010.108).

13 Kieser, T., Bibb, M. J., Buttner, M. J., Chater, K. F. \& Hopwood, D. A. Practical Streptomyces Genetics, Ch. 22 (The John Innes Foundation, Norwich, 2000).

14 Hopwood, D. A. et al. Genetic Manipulation of Streptomyces: A Laboratory Manual ( 1985).

15 Wong, L. L. Cytochrome P450 monooxygenases. Curr. Opin. Chem. Biol. 2, 263-268 (1998).

16 Sasaki, J., Mikami, A., Mizoue, K. \& Omura, S. Transformation of 25- and 1 alphahydroxyvitamin D3 to 1 alpha, 25-dihydroxyvitamin D3 by using Streptomyces sp. strains. Appl. Environ. Microbiol. 57, 2841-2846 (1991).

17 Taylor, M., Lamb, D. C., Cannell, R., Dawson, M. \& Kelly, S. L. Cytochorme P450105D1 (CYP105D1) from Streptomyces griseus: heterologous expression, activity, and activation effects of multiple xenobiotics. Biochem. Biophys. Res. Commun. 263, 838-842 (1999).

18 Sawada, N. et al. Conversion of vitamin D3 to 1alpha, 25-dihydroxyvitamin D3 by Streptomyces griseolus cytochrome P450SU-1. Biochem. Biophys. Res. Commun. 320, 156-164 (2004)

19 Chang, Y. T. \& Loew, G. H. Construction and evaluation of a three-dimensional structure of cytochrome P450choP enzyme (CYP105C1). Protein Eng. 9, 755-766 (1996).

20 Waterman, M., Johnson, E., Abelson, J. \& Simon, M. Cytochrome P450. Methods Enzymol 206, 3-11 (1991).

21 Kieser, T., Bibb, M. J., Buttner, M. J., Chater, K. F. \& Hopwood, D. A. Practical Streptomyces Genetics, Ch. 9 (The John Innes Foundation, Norwich, 2000).

22 Jennewein, S. et al. Coexpression in yeast of Taxus cytochrome P450 reductase with cytochrome P450 oxygenases involved in Taxol biosynthesis. Biotechnol. Bioeng. 89, 588-598 (2005).

23 Zhan, J. X. et al. Microbial metabolism of Artemisinin by Mucor polymorphosporus and Aspergillus niger. J. Nat. Prod. 65, 1693-1695 (2002).

24 Sandstrom, P., Welch, W. H., Blomquist, G. J. \& Tittiger, C. Functional expression of a bark beetle cytochrome P450 that hydroxylates myrcene to ipsdienol. Insect Biochem. Mol. Biol. 36, 835-845 (2006).

25 Lamb, D. C. et al. Cytochrome P450 complement (CYPome) of the avermectinproducer Streptomyces avermitilis and comparison to that of Streptomyces coelicolor A3 (2). Biochem. Biophys. Res. Commun. 307, 610-619 (2003).

26 Lamb, D. C. et al. The cytochrome P450 complement (CYPome) of Streptomyces coelicolor A3 (2). J. Biol. Chem. 277, 24000-24005 (2002).

27 Takano, E., White, J., Thompson, C. J. \& Bibb, M. J. Construction of thiostreptoninducible high-copy-number expression vectors for use in Streptomyces spp. Gene 166, 133-137 (1995).

28 Ueno, M., Yamashita, M., Hashimoto, M., Hino, M. \& Fujie, A. Oxidative activities of heterologously expressed CYP107B1 and CYP105D1 in whole-cell biotransformation using Streptomyces lividans TK24. J Biosci. Bioeng. 100, 567-572 (2005).

29 Hussain, H. A. \& Ward, J. M. Enhanced heterologous expression of two Streptomyces griseolus cytochrome P450s and Streptomyces coelicolor ferredoxin reductase as potentially efficient hydroxylation catalysts. Appl. Environ. Microbiol. 69, 373-382 (2003).

30 Herai, S. et al. Hyper-inducible expression system for streptomycetes. Proc. Natl Acad. Sci. USA 101, 14031-14035 (2004). 